\title{
Mise en évidence des effets d'une irradiation aiguë localisée par tomodensitométrie et scintigraphie au gallium-67 : approche dans un modèle expérimental
}

\author{
J.-L. ALBÉRINI*, P. BOURGUET*, L. DAZORD*, J.-L. LEFAIX**
}

(Manuscrit reçu le 20 juin 1996, révisé le 27 octobre 1996, accepté le 2 décembre 1996)

RÉSUMÉ Récemment encore, les méthodes d'imagerie proposées dans le diagnostic précoce des lésions d'irradiation localisée semblaient faire défaut. La tomodensitométrie et la scintigraphie au gallium-67, examens de pratique courante, ont été étudiées dans un modèle expérimental d'irradiation localisée, développé après plusieurs accidents de surexposition. Douze lapins ont été irradiés par une source d'Iridium-192, avec une dose unique de $120 \mathrm{~Gy}$ à la peau délivrée en regard du muscle iliospinal droit, au niveau de la cinquième vertèbre lombaire. Des modifications précoces ont été observées dans ce modèle expérimental par ces 2 méthodes d'imagerie in vivo, avec une hyperfixation scintigraphique en regard de la lésion d'irradiation dès le troisième jour après irradiation et des anomalies tomodensitométriques évolutives au cours du premier mois, avec des résultats homogènes dans toute la cohorte. Ces 2 techniques pourraient contribuer à un diagnostic précoce et une évaluation pronostique des lésions d'irradiation aiguë. Un suivi portant sur une durée de 4 mois a permis d'envisager la poursuite de cette étude pour une durée de 18 à 24 mois, permettant d'aborder les processus tardifs, classiquement caractérisés par des délabrements musculaires et le développement d'une sclérose atrophique.

ABSTRACT Imaging of acute irradiation lesions in experimental model : approach by Gallium-67 and X-rays computed tomography (CT).

Actually, imaging for diagnosis of acute localized irradiation lesions are lacking. CT and ${ }^{67} \mathrm{Ga}$ scintiscans were studied in an experimental model of acute localized irradiations developed after several accidental overexposures. Irradiation with an industrial radiographic source (Iridium 192) was used in this study. Twelve rabbits were irradiated on the back on the ilio-spinalis muscle (Vth lumbar vertebra level) at a skin surface dose of $120 \mathrm{~Gy}$. Early modifications were observed in this model : an uptake in scintigraphy at the level of the irradiation lesion three days after irradiation and evolutive CT abnormalities during the first month. These both imaging techniques could be proposed for an early diagnosis, an evaluation of early irradiation injuries and help the decision of the clinician. Because a follow-up for 4 months is not long enough, the delayed process (atrophic sclerosis, fibrosis, muscular alterations) will be studied for 18-24 months after irradiation.

\footnotetext{
* Service de Médecine Nucléaire, Centre Eugène Marquis, rue de la Bataille FlandresDunkerque, 35062 Rennes Cedex, France

** CEA-DSV, Laboratoire de Radiobiologie Appliquée, CEN-Saclay, 91191 Gif-sur-Yvette Cedex, France
} 


\section{Introduction}

Les lésions d'irradiation aiguë sont rencontrées dans le cadre des accidents humains de surexposition.

Les manifestations cliniques de ce type d'accident peuvent être retardées et apparaître avec une évolution en plusieurs phases. Elles sont extériorisées sous forme de lésions cutanées, qui ne permettent pas de présumer du degré de gravité de l'atteinte radiologique, ni d'évaluer l'extension des lésions sousjacentes. L'évolution pronostique des lésions est donc souvent difficile à établir et peut aboutir à des processus fibronécrotiques destructifs associés à des phénomènes inflammatoires.

Compte tenu de la faiblesse des moyens d'investigation et d'évaluation des lésions d'irradiation localisées proposées aux cliniciens amenés à prendre en charge des patients accidentés, il nous a semblé intéressant d'étudier deux techniques d'imagerie non-invasives, qui pourraient aider la décision thérapeutique. Celles-ci doivent obéir à un certain nombre de critères, représentant les qualités principales demandées aux méthodes biophysiques, à savoir permettre:

1) un diagnostic précoce et une localisation précise des lésions,

2) une évaluation de la sévérité des lésions, reflet de l'intensité de l'atteinte radiologique,

3) un suivi de l'efficacité du traitement médico-chirurgical,

4) un suivi après guérison, en envisageant une possibilité de récidive de lésions tardives.

Dans cette étude, la tomodensitométrie et la scintigraphie au ${ }^{67} \mathrm{Ga}$ ont été employées non pas dans un but biophysique d'évaluation dosimétrique, mais avec deux objectifs :

- d'une part, identifier le plus précocement possible des lésions déterminantes pour le pronostic et l'évolution des tissus irradiés, notamment pour les tissus situés en profondeur,

- d'autre part, envisager de suivre à plus long terme ces lésions classiquement caractérisées par des processus tardifs fibrotiques, présents à partir du $4^{\circ}$ mois dans le modèle expérimental animal utilisé.

Ce modèle simulant une irradiation localisée rencontrée dans des accidents de surexposition (Lefaix et al., 1992b, 1993b) présente des ressemblances avec la radiopathologie humaine. Il a déjà été utilisé pour apporter des informations diagnostiques et thérapeutiques (Lefaix et al., 1992b). Les lésions ont été provoquées chez des lapins par une source collimatée d'iridium 192 (192 Ir), en une exposition unique de 120 Gy à la peau, à fort débit de dose, évalué à $16 \mathrm{~Gy} / \mathrm{min}$.

Ce modèle homogène et standardisé possédait 2 avantages : il permettait d'aborder la phase précoce après irradiation, avec la présence de lésions ren- 
contrées dans une situation accidentelle, mais également de suivre le développement de la fibrose musculaire, au stade tardif. En effet, la fibrose, définie par une infiltration fibroblastique intense et un dépôt excessif de matrice extracellulaire (Delanian, 1994), est un processus dynamique et évolutif. Elle représente le terme ultime d'un processus inflammatoire chronique.

L'objectif pour la scintigraphie était d'apprécier l'importance des phénomènes d'inflammation présents au niveau des tissus lésés et pouvant jouer un rôle dans l'évolution vers des processus fibronécrotiques, car le ${ }^{67} \mathrm{Ga}$, isotope à tropisme cellulaire, a la propriété de s'accumuler dans les lésions inflammatoires, suivant plusieurs mécanismes (Ando et al., 1990; Bitar et al., 1986; Camargo et al., 1979 ; Hoffer et al., 1977, 1980 ; Menon et al., 1978 ; Tsan, 1978, 1985 ; Weiner et al., 1981).

La tomodensitométrie devait jouer un rôle complémentaire en étudiant les lésions affectant les tissus profonds. Ces 2 techniques fonctionnelle pour la scintigraphie et morphologique pour la tomodensitométrie permettent une imagerie in vivo. Le suivi porte sur plus de 4 mois, mais nous rapportons ici les résultats observés au cours du premier mois, délai critique dans le cadre des accidents de surexposition, notamment pour la prise en charge des accidentés (Daburon et al., 1986).

\section{Matériels et méthodes}

\subsection{Irradiation}

L'expérimentation réalisée bénéficie de l'autorisation No. 3255 de l'Office de protection animale du Ministère de l'agriculture et de la forêt. Le modèle expérimental utilisé a été développé au Laboratoire de radiobiologie appliquée de Jouy-en-Josas.

Douze femelles lapin de race New Zealand, pesant de 3 à $3,5 \mathrm{~kg}$, ont reçu une dose unique d'irradiation de 120 Gy à la peau au moyen d'une source scellée cylindrique d'Iridium 192 (émetteur $\gamma$ d'énergie moyenne de $380 \mathrm{keV}$, avec les principales émissions à 296, 308, 316 et $468 \mathrm{keV}$ ), placée dans un appareil de gammagraphie industrielle (CGR de type GR-50).

Le débit de dose est de $16 \mathrm{~Gy} \mathrm{~min}^{-1}$ à la peau. Le faisceau d'irradiation conique d'un angle solide de $60^{\circ}$ affecte les tissus cutanés, sous-cutanés et le muscle sous-jacent, en l'occurrence le muscle ilio-spinal droit. Le collimateur est placé au contact de la peau, sans pression, à $3 \mathrm{~cm}$ à droite de la ligne médiane, en regard de la cinquième vertèbre lombaire.

Les animaux sont anesthésiés avec une association de kétamine (Imalgène $1000^{\circledR}$, Rhône Poulenc - $50 \mathrm{mg} \mathrm{kg}^{-1}$ ) et d'acépromazine (Vétranquil ${ }^{\circledR}$, Clin May - $5 \mathrm{mg} \mathrm{kg}^{-1}$ ) injectée par voie sous-cutanée en une seule administration. Ce protocole sera identique pour l'acquisition des images scintigraphiques et 
tomodensitométriques. Lors de précédents travaux (Lefaix et al., 1992a, 1993b), une étude dosimétrique a été réalisée. La dose délivrée à la peau est normalisée à $100 \%$. Les doses absorbées à $1,2,3$ et $4 \mathrm{~cm}$ de profondeur correspondent respectivement à 46, 25, 15 et $10 \%$ de la dose à la peau (Lefaix et al., 1992a, 1993b). Au niveau du site d'irradiation, le muscle ilio-spinal est situé à une profondeur de $0,5 \mathrm{~cm}$ et possède une épaisseur de $2 \mathrm{~cm}$ environ.

\subsection{Animaux}

Les animaux sont logés séparément dans des cages en métal, équipées de rouleaux de papiers permettant le recueil quotidien des urines et fèces. Ils sont soumis à un cycle de 12 heures de lumière artificielle et de 12 heures d'obscurité. Les urines sont évacuées par une bonde d'évacuation, vers des cuves de décroissance. Les fèces sont des déchets radioactifs et sont placées dans des sacs plastiques dans l'aire de stockage de décroissance des déchets produits par le service de médecine nucléaire.

\subsection{Acquisition des images}

\subsubsection{Scintigraphie}

Le ${ }^{67} \mathrm{Ga}$ est un radio-isotope de 78,3 heures de période, du groupe 3 . Les principales émissions sont de type $\gamma$ de 93,185 et $300 \mathrm{keV}$ et des électrons de 8, 84, $93 \mathrm{keV}$. Une dose de $25 \mathrm{MBq}$ de ${ }^{67} \mathrm{Ga}$ (Mallinckrodt Diagnostica, France) est administrée en bolus par voie intraveineuse dans une veine marginale de l'oreille. Un délai de 2 semaines au minimum a été respecté entre 2 injections pratiquées pour chaque animal. Ce délai permet d'estimer la dose résiduelle en rapport avec l'injection précédente à moins de $5 \%$ (en prenant en considération la décroissance radioactive, à laquelle il faudrait rajouter l'élimination urinaire et fécale).

Quarante-huit heures après l'injection, les images planaires et tomographiques sont obtenues avec une gamma caméra grand champ (Apex View AG - Elscint) équipée d'un collimateur à trous parallèles moyenne énergie, moyenne résolution et connectée à un système informatique (Sophy NXT Sopha Medical).

La spectroscopie est réglée sur les pics de 93 et $185 \mathrm{keV}$ avec une fenêtre de $10 \%$. Les animaux sont placés sur le ventre dans une gouttière, permettant un positionnement reproductible pour les différents animaux. Une image en vue postérieure (centrée sur la lésion cutanée) acquise en prétemps de 600 secondes dans une matrice de $256 \times 256$ avec un zoom de 1,66 (Fig. 1), est couplée à une image avec repérage de la lésion cutanée grâce à un crayon au Cobalt 57 (dont le pic d'absorption totale est de $122 \mathrm{keV}$ ).

L'acquisition tomoscintigraphique est pratiquée selon un mode pas à pas avec un zoom de 2,00. Trente-deux images de 30 secondes chacune sont réali- 


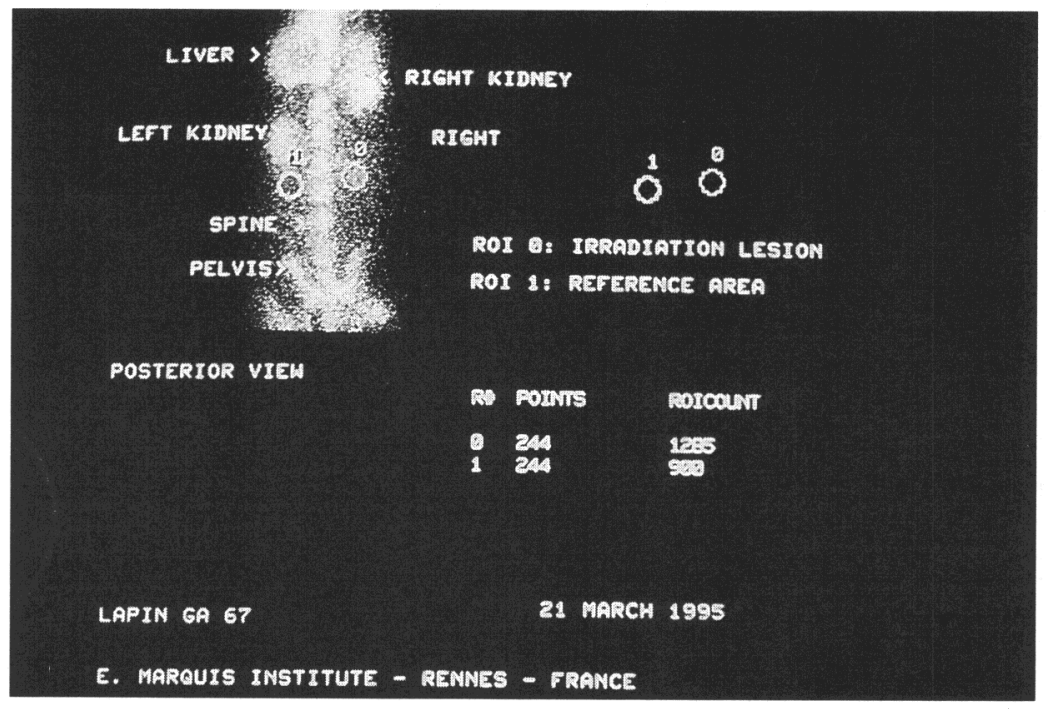

Fig. 1. - Semaine 4. Scintigraphie réalisée en incidence postérieure (en haut à gauche) permettant de visualiser les structures osseuses (bassin, rachis), les reins et la lésion hyperfixante sous le pôle inférieur du rein droit, avec les régions d'intérêt. (ROI $)$ est placée autour de l'hyperfixation lésionnelle, à droite et à une distance d du rachis, $\left(\mathrm{ROI}_{1}\right)$ est placée du côté controlatéral, à la même distance d du rachis que la $\mathrm{ROI}_{0}$. Le nombre de coups dans les deux régions d'intérêt $\left(\mathrm{ROI}_{0}\right.$ et $\left.\mathrm{ROI}_{1}\right)$ est indiqué en bas à droite.

Week 4. Scintiscan posterior view (on the left above) with osseous structures (pelvis, spine), kidneys and uptake increase under the right kidney, with regions of interest. $\left(\mathrm{ROI}_{0}\right)$ is determined around the uptake of the lesion, to the right of spine. $\left(\mathrm{ROI}_{1}\right)$ is determined on the left side of spine, with the same distance from spine than $\left(\mathrm{ROI}_{0}\right)$. The count rates in the regions of interest are indicated on the right below.

sées sur un arc de $180^{\circ}$ et stockées dans une matrice de 64 × 64. Les données brutes stockées sur disque optique sont reconstruites par rétroprojection filtrée avec un filtre de type Hanning.

\subsubsection{Tomodensitométrie}

Les coupes scanographiques réalisées à l'aide d'un appareil CT Pace (General Electric Milwaukee) sont centrées sur le rachis et les muscles paravertébraux. Les coupes jointives d'une largeur de $5 \mathrm{~mm}$ sont réalisées avec les critères d'acquisition suivant: $120 \mathrm{kV}, 80$ ou $100 \mathrm{~mA}, 3$ secondes par coupe, avec un filtre standard. Le champ d'acquisition est de $25 \mathrm{~cm}$ et le champ de reconstruction de $10 \mathrm{~cm}$. 


\subsection{Analyse des données}

\subsubsection{Scintigraphie}

La réalisation d'une étude préliminaire chez deux lapins a permis d'une part de déterminer le délai idéal entre l'injection du ${ }^{67} \mathrm{Ga}$ et l'acquisition des clichés, puis le délai minimum entre deux injections, et d'autre part de montrer qu'il y a chez les animaux sains une accumulation $\mathrm{du}{ }^{67} \mathrm{Ga}$ symétrique et comparable en regard des muscles ilio-spinaux droit et gauche. Nous avons donc utilisé une méthode semi-quantitative pour le calcul de l'intensité de l'hyperfixation présente en regard de la lésion d'irradiation. Deux régions d'intérêt (ROI) circulaires de taille identique (244 pixels) sont placées sur l'image planaire réalisée en incidence postérieure (Fig. 1) :

- la première aire, $\mathrm{ROI}_{0}$, placée autour de l'hyperfixation présente en regard de la lésion, à une distance $d$ du rachis,

- la deuxième aire, $\mathrm{ROI}_{1}$, placée à gauche du rachis à la même distance $d$ du rachis que la $\mathrm{ROI}_{0}$.

La $\mathrm{ROI}_{1}$ est prise comme valeur de référence de la fixation moyenne.

La fixation de l'aire irradiée est comparée à celle de l'aire de référence avec le rapport: nombre de coups dans l'aire $0 /$ (somme du nombre de coups dans l'aire 0 et dans l'aire 1) (Fig. 1). Ce rapport est nommé : $\mathrm{ROI}_{0}\left(\mathrm{ROI}_{0}+\mathrm{ROI}_{1}\right)$. Il est égal à 0,5 lors de l'absence de fixation, observée chez des animaux sains.

Les études de la biodistribution ont été réalisées afin de vérifier que les foyers d'accumulation du ${ }^{67} \mathrm{Ga}$ concordaient avec les anomalies scintigraphiques. Des prélèvements de la lésion cutanée, de peau saine, de la lésion musculaire - repérée par tomodensitométrie préalablement - et de muscle sain ont été pratiqués. Ces prélèvements de tissus, comme les reins, le foie, la rate et le sang, constituent des points de repère pour apprécier le niveau d'accumulation du ${ }^{67} \mathrm{Ga}$ dans la peau et le muscle. Les comptages d'activité des prélèvements de fragments tissulaires, pesés et placés dans des tubes, sont réalisés dans un appareil passeur d'échantillons $\gamma$ Kontron ${ }^{(B)}$.

Les valeurs sont exprimées en pourcentage de la dose injectée par gramme de tissu $\times 10^{-3}$. Les comptages d'activité sont étalonnés à partir d'un échantillon d'un millilitre d'une solution contenant une faible activité diluée dans $500 \mathrm{ml}$ d'eau (Fig. 2). Le calcul est le suivant :

nombre de coups par gramme de tissu $\times$ activité de la solution $\times 100$

nombre de coups dans $1 \mathrm{ml}$ de solution $\times 500 \mathrm{ml} \times$ activité injectée

\subsubsection{Tomodensitométrie}

La taille des lésions, leur densité, sans et avec injection de produit de contraste (Ioxitalamate de sodium et de Méglumine, Télébrix $35^{\circledR}$ ) ont été mesurées sur les coupes. Il a également été possible de comparer les surfaces des muscles ilio-spinaux droit et gauche, afin de quantifier l'atrophie du muscle droit par 
MISE EN ÉVIDENCE DES EFFETS D'UNE IRRADIATION AIGUË LOCALISÉE

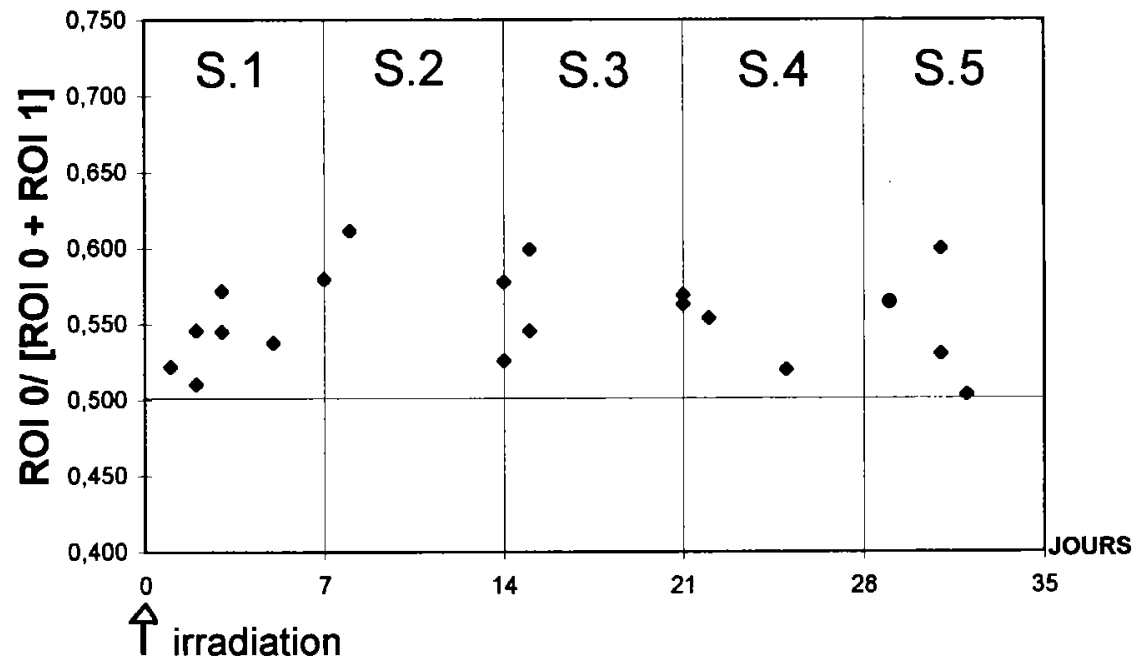

Fig. 2. - Scintigraphie. Évolution en fonction du temps du rapport du nombre de coups des régions d'intérêt $\mathrm{ROI}_{0} /\left(\mathrm{ROI}_{0}+\mathrm{ROI}_{1}\right)$.

Scintigraphy. Time ratio curve $\mathrm{ROI}_{0} /\left(\mathrm{ROI}_{0}+\mathrm{ROI}_{I}\right)$.

rapport au gauche, en délimitant les bords des muscles et en mesurant leur surface. Les mesures de surface des muscles ont été réalisées sur la coupe passant au milieu de l'hypodensité du muscle ilio-spinal, ainsi que sur les 3 coupes audessus et au-dessous de celle-ci. Puis, nous avons calculé la moyenne du rapport des surfaces des muscles droit/gauche, sur ces 7 coupes.

\section{Résultats}

Les modifications observées étaient datées par rapport à la semaine $\left(\mathrm{S}_{0}\right)$ d'irradiation. Elles possédaient un caractère évolutif pour un suivi allant de $S_{0}$ à $S_{21}$.

\subsection{Scintigraphie}

Elle est apparue perturbée précocément, puisqu'une hyperfixation est notée du côté irradié dès le 3ème jour après l'irradiation. L'hyperfixation apparaît modérée et stable pendant le premier mois après l'irradiation. Sur les images planaires, cette hyperfixation a été visualisée en regard de la lésion d'irradiation, mais la détermination du rapport $\mathrm{ROI}_{0} /\left(\mathrm{ROI}_{0}+\mathrm{ROI}_{1}\right)$ permettait une description semi-quantitative de l'intensité de l'accumulation du ${ }^{67} \mathrm{Ga}$ par la lésion d'irradiation (Fig. 2). La moyenne de ce rapport est voisine de 0,55 $+/-0,04$, durant le premier mois. Cette valeur est significativement différente de 0,5 , d'après l'analyse statistique (test de Man-Whitney et test de $\chi^{2}$ ). 
Les résultats scintigraphiques ont pu être confirmés lors de deux premières études de biodistribution réalisées lors de la mort accidentelle d'animaux, 48 heures après l'injection de ${ }^{67} \mathrm{Ga}, 15$ et 28 jours après l'irradiation $\left(S_{2}, S_{4}\right)$. Il existe en effet une accumulation de ${ }^{67} \mathrm{Ga}$ dans le muscle irradié et dans la lésion cutanée (Tableau I).

En revanche, l'analyse de la distribution du ${ }^{67} \mathrm{Ga}$ dans les tissus situés en profondeur n'est pas possible sur les données tomoscintigraphiques, du fait du manque de résolution spatiale et de la présence d'artéfacts de reconstruction en rapport avec la proximité du rein droit.

TABLEAU I

\section{Résultats des études de biodistribution. Les valeurs sont exprimées en pourcentage de la dose injectée/g de tissu $\times 10^{-3}$ Results of biodistribution studies. Values in percent of injected dose (ID)/g of tissue $\times 10^{-3}$}

\begin{tabular}{|lrr|}
\hline Detai apres irradiation & $\mathbf{J}_{\mathbf{1 5}}$ & $\mathbf{J}$ \\
Déat apres tujection & $\mathbf{4 8 ~ \mathbf { ~ h }}$ & $\mathbf{4 8 \mathbf { h }}$ \\
Peau irradiée & 46,80 & 15,70 \\
Peau saine & 6,95 & 4,80 \\
Muscle irradié & 0,80 & 0,70 \\
Muscle sain & 0,77 & 0,79 \\
Foie & 54,27 & 78,90 \\
Rate & 128,60 & 52,48 \\
Rein droit & 128,20 & 245,00 \\
Rein gauche & 113,50 & 214,50 \\
Sang & 22,75 & 22,10 \\
\hline
\end{tabular}

\subsection{Tomodensitométrie}

Un épaississement cutané est noté dès la première semaine $\left(\mathrm{S}_{1}\right.$, Fig. 3), comparativement à l'autre côté, alors que la densité du revêtement cutané à l'endroit de cet épaississement est plus élevée que celle de la peau saine (70 à 90 unités Hounsfield (UH) vs. 25 UH).

Durant la deuxième semaine $\left(\mathrm{S}_{2}\right)$, une modification des tissus sous-cutanés est observée: la graisse disparait et est remplacée par une formation hypodense par rapport au muscle sous-jacent, à savoir le muscle ilio-spinal droit (40 UH vs. 70 UH, Fig. 4). L'épaisseur de la lésion était comprise entre 2 ou $3 \mathrm{~mm}$ et le diamètre entre 10 et $20 \mathrm{~mm}$. Cette formation est inconstante. 


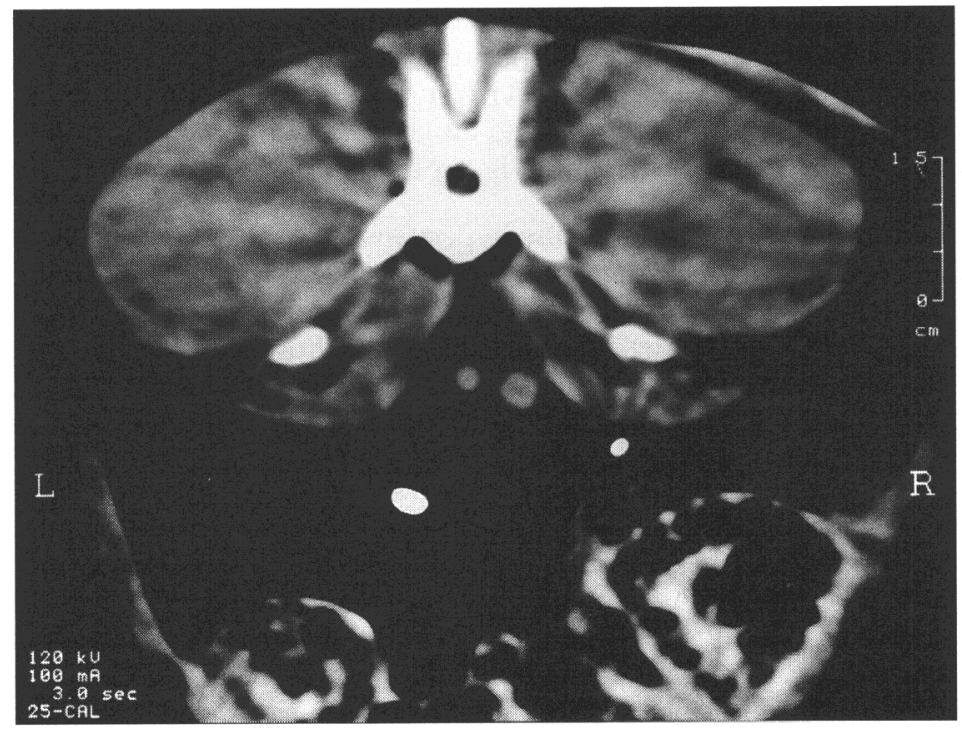

Fig. 3. - Semaine 1. Tomodensitométrie. Epaississement et hyperdensité du plan cutané à droite.

Week 1. CT. Thickening and cutaneous hyperdensity.

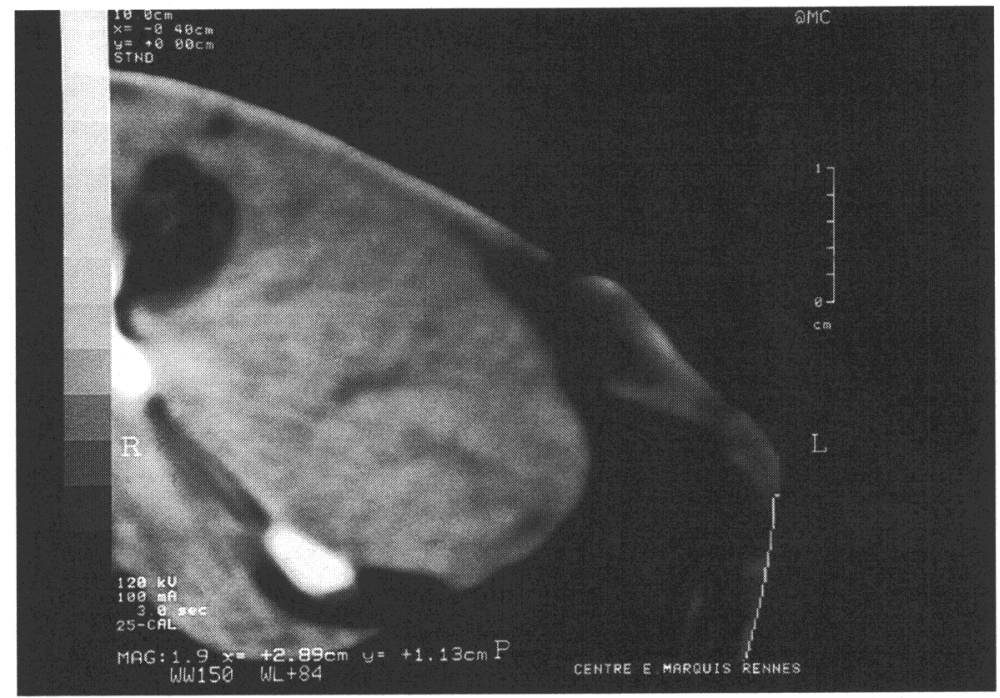

Fig. 4. - Semaine 2. Tomodensitométrie. Hypodensité sous-cutanée.

Week 2. CT. Hypodense lesion in the subcutaneous tissues. 
À la troisième semaine $\left(\mathrm{S}_{3}\right)$, une lésion hypodense (40 UH environ contre $70 \mathrm{UH}$ pour le muscle sain) est apparue à la superficie du muscle ilio-spinal droit, associée ou non à la lésion décrite précédemment (Fig. 5). Son volume estimé de forme ellipsoïde a progressé au cours du temps, à partir de $S_{3}$, avec une progression de la superficie vers la profondeur.

À la quatrième semaine $\left(\mathrm{S}_{4}\right)$, une hyperdensité localisée au bord externe du muscle ilio-spinal droit, siégeant à peu près au milieu de l'hypodensité décrite précédemment, est observée : sa densité est voisine de $65 \mathrm{UH}$ au départ et augmente jusqu'à 120 UH (Fig. 6). Des clichés radiographiques de biopsie de lésion musculaire réalisés avec des rayons $X$ de basse énergie montrent que cette hyperdensité correspond à des dépôts calciques. Aucun rehaussement après injection de produit de contraste n'est noté.

Il n'a pas été observé d'atrophie du muscle ilio-spinal droit avant $\mathrm{S}_{6}$, date à partir de laquelle l'hypodensité du muscle ilio-spinal droit est devenue hétérogène, constituée d'une couronne de densité comprise entre 35 et $40 \mathrm{UH}$ et de plages centrales plus hypodenses (15 à $20 \mathrm{UH}$ ) qui ont conflué au cours du temps. Ces plages pourraient correspondre à de la nécrose, d'après les résultats des précédentes études histopathologiques (Brocheriou et al., 1986; Lefaix et al., 1993b).

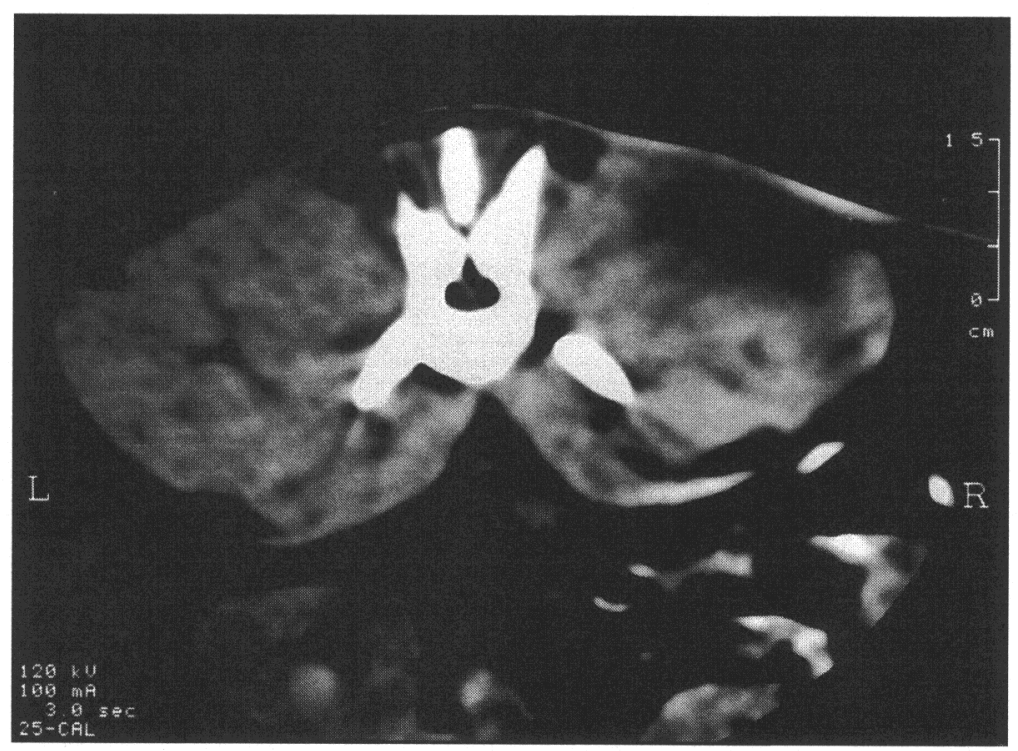

Fig. 5. - Semaine 3. Tomodensitométrie. Hypodensité du plan musculaire superficiel (nécrose).

Week 3. CT. Hypodensity in a superficial zone of the right ilio-spinalis muscle. 


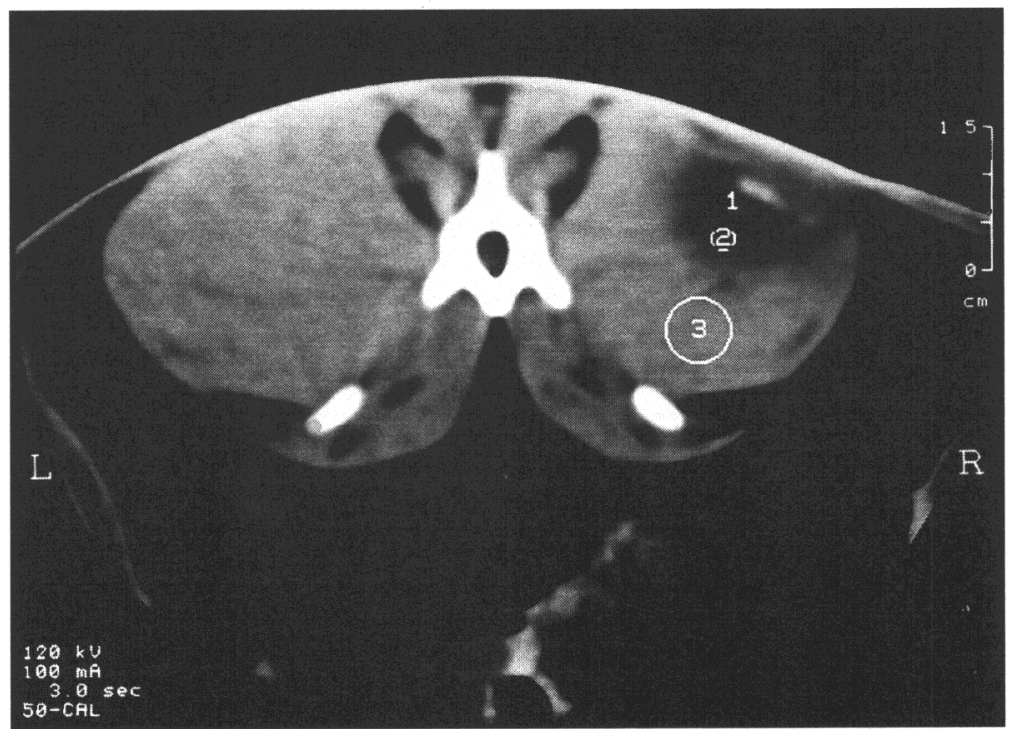

Fig. 6. - Semaine 4. Tomodensitométrie. Hyperdensité en rapport avec une calcification (région 1 de densité de $120 \mathrm{UH}$ ) et hypodensité (région 2 de 20 à $40 \mathrm{UH}$ ) du plan musculaire superficiel. La densité normale du muscle est de $70 \mathrm{UH}$ (zone 3).

Week 4. CT. Hyperdensity $\left(\mathrm{ROI}_{1}=120 \mathrm{UH}\right)$ and hypodensity $\left(\mathrm{ROI}_{2}=20\right.$ $40 \mathrm{UH}$ ) in a superficial area of the muscle.

\section{Discussion}

Depuis 25 ans, plusieurs accidents sont survenus à la suite de contacts fortuits avec des sources scellées, utilisées pour des usages industriels et médicaux, comme le ${ }^{60} \mathrm{Co}, \mathrm{l}^{192} \mathrm{Ir}$, et les rayons X (Lefaix et al., 1992a, b, 1993b).

Ce type d'accident est à l'origine de graves lésions qui peuvent intéresser des volumes tissulaires considérables et entraîner des complications redoutables: séquelles fonctionnelles ou esthétiques, syndrôme algique, et nécessitent une prise en charge thérapeutique rapide (Daburon et al., 1986 ; Nénot, 1990). Les régions affectées sont surtout les mains, les cuisses et le thorax (Lefaix et al., 1992b).

Peu de méthodes ont été proposées jusqu'à ce jour dans un but diagnostique précoce. Il n'a pas été rapporté d'études par tomodensitométrie ou scintigraphie au ${ }^{67} \mathrm{Ga}$ dans les lésions d'irradiation aiguë.

D'autres techniques ont été proposées, comme la thermographie (Daburon, 1986), mais ces techniques ne sont plus répandues. Des études par IRM ont été 


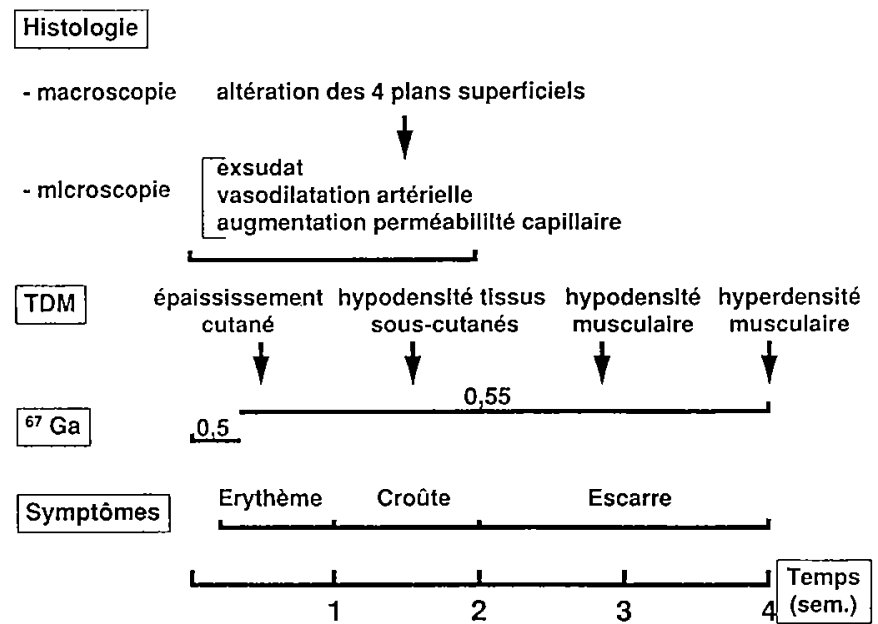

Fig. 7. - Corrélation des résultats scintigraphiques, tomodensitométriques et histopathologiques.

Correlation for scintigraphic, CT results and histopathologic findings.

réalisées (Chalansonnet et al., 1993) et sont en cours. Il faut signaler également que d'autres techniques isotopiques ont été expérimentées dans les lésions d'irradiation localisées : la scintigraphie vasculaire au 99m Tc (Raina et al., 1992) et la scintigraphie au ${ }^{201} \mathrm{Tl}$, permettant d'apprécier l'étendue de la nécrose sur des animaux sacrifiés (Hoffschir et al., 1989).

Le modèle lapin a été choisi pour la réalisation de ce travail, du fait de la commodité qu'il présentait pour la réalisation des deux techniques d'imagerie. Il est également étudié actuellement par imagerie par résonance magnétique, à l'hôpital $\mathrm{H}$. Mondor de Créteil. Les résultats de la tomodensitométrie et de la scintigraphie au ${ }^{67} \mathrm{Ga}$ pourront ainsi être confrontés à ceux de l'IRM. La petite taille de l'animal a constitué toutefois un handicap pour les études tomoscintigraphiques, puisque seules des images planaires ont pu être analysées.

La principale faiblesse de la scintigraphie est son manque de précision pour localiser la lésion d'irradiation en profondeur, mais le volume de tissu musculaire concerné par l'inflammation pourrait être mieux précisé chez l'homme par des tomoscintigraphies, réalisées actuellement dans les services de Médecine nucléaire dans des indications de pathologies néoplasiques, pour préciser la topographie d'une hyperfixation visualisée éventuellement sur une image planaire.

Ce modèle animal a précédemment permis l'évaluation de l'efficacité de traitements pharmacologiques, avec des associations médicamenteuses mon- 
trant que le bénéfice était le plus significatif après administration d'anti-inflammatoires. Une efficacité maximale est reconnue pour l'association d'un antiinflammatoire non-stéroïdien, le flurbiprofène avec un vasodilatateur, la trimétazidine (Lefaix et al., 1992b). Le résultat obtenu est analogue à un abaissement de la dose d'irradiation d'un facteur 2, à la fois sur l'intensité et l'évolution de la lésion au plan cutané et sur l'étendue en profondeur du délabrement musculaire.

La confrontation des résultats scintigraphiques et tomodensitométriques avec les données histopathologiques permet d'expliquer la sémiologie tomodensitométrique et scintigraphique (Fig. 7).

Il est intéressant de présenter la description de l'évolution radiopathologique du modèle lapin avec la chronologie des lésions cliniques et des anomalies histopathologiques correspondantes (Brocheriou et al., 1986; Lefaix et al., 1985, 1992b, 1993b; Verola et al., 1986).

Cliniquement, pour une dose unique de $120 \mathrm{~Gy}$ à la peau, un érythème et

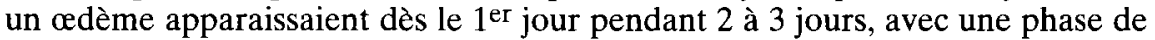
latence entre le 4ème et le 6ème jour. A partir du 11ème jour, une ulcération superficielle entourée d'une couronne érythémateuse était observée, remplacée par une épithélite exsudative vers le 30 ème jour. Le remplacement de cette zone par du tissu bourgeonnant amenait à la cicatrisation cutanée entre le quatrième et le sixième mois.

L'évolution histopathologique a montré 4 phases principales (Lefaix et al., 1992b ; Verola et al., 1986) :

1) phase avec macroscopiquement une nécrose du derme avec ulcère cutané adhérent aux tissus sous-jacents avant $S_{5}$, alors que microscopiquement était observée une atteinte musculaire avec une congestion et un exsudat, témoins d'une augmentation du flux vasculaire et d'une augmentation de la perméabilité capillaire, ainsi que la présence de microthrombi.

2) phase avec une réaction inflammatoire du plan musculaire superficiel avant $\mathrm{S}_{4}$, et du plan musculaire profond avant $\mathrm{S}_{7}$, et une atteinte du tissu conjonctivovasculaire. Après $S_{4}$, une nécrose fibrinoïde des parois vasculaires, un infiltrat inflammatoire périvasculaire et des parois artériolaires, ainsi que des thrombi fibrineux étaient notés.

3) phase avec une radionécrose ischémique des plans musculaires superficiel et profond, associés à un infiltrat de polynucléaires et quelques lésions vasculaires minimes après $S_{10}$, avec la confluence de foyers de nécrose, en rapport avec des altérations vasculaires en profondeur et une fibrose interstitielle.

4) phase, tardive, avec une sclérose atrophique du plan musculaire superficiel avant $S_{20}$ et du plan musculaire profond avant $S_{30}$.

Nous présentons les quatre hypothèses émises par différentes équipes pour expliquer les mécanismes d'accumulation du ${ }^{67} \mathrm{Ga}$ dans les lésions inflamma- 
toires, permettant de comprendre l'accumulation $\mathrm{du}{ }^{67} \mathrm{Ga}$ au niveau de la lésion d'irradiation:

1) fixation bactérienne directe (Hoffer, 1980; Menon, 1978; Tsan, 1978b, 1985),

2) augmentation de la perméabilité vasculaire (Tsan, 1985),

3) mécanismes indirects concernant les polynucléaires (Ando, 1990; Bitar, 1986 ; Camargo, 1979 ; Hoffer et al., 1977, 1980 ; Tsan et al., 1978a, b ; Weiner et al., 1981),

4) fixation directe par les leucocytes ou autre élément figuré du sang (Camargo et al., 1979; Gelrud et al., 1974; Hoffer, 1980 ; Tsan et al., 1978a, b, 1985 ; Weiner et al., 1981).

Les trois dernières hypothèses permettent d'expliquer l'accumulation de ${ }^{67} \mathrm{Ga}$ dans la lésion d'irradiation.

La deuxième hypothèse est retenue, car les études histopathologiques ont constaté un exsudat important dès 24 heures après l'irradiation, en rapport avec une augmentation de la perméabilité, ce qui permettrait d'expliquer la présence d'une fixation du ${ }^{67} \mathrm{Ga}$ apparente dès le troisième jour après irradiation.

La troisième hypothèse repose sur un mécanisme de transport du ${ }^{67} \mathrm{Ga}$ par les leucocytes (Ando et al., 1990 ; Bitar et al., 1986) vers le foyer inflammatoire, grâce au chimiotactisme (Camargo et al., 1979; Tsan et al., 1978a).

La quatrième hypothèse est souvent retenue comme la plus probable : elle fait appel à un transport par différentes protéines plasmatiques, comme la lactoferrine (Hoffer et al., 1977, 1980 ; Weiner et al., 1981), la transferrine (Hoffer et al., 1977, 1980), la ferritine ou des sidérophores (Tsan, 1978b, 1985), vers le site inflammatoire avec relargage sur la lactoferrine contenue dans les leucocytes (Gelrud et al., 1974 ; Hoffer et al., 1980 ; Weiner et al., 1981). En histopathologie, un infiltrat inflammatoire est noté avant $\mathrm{S}_{4}$, permettant d'expliquer l'accumulation du ${ }^{67} \mathrm{Ga}$ dans les tissus, notamment musculaires.

L'hyperfixation scintigraphique peut donc être expliquée par l'exsudat précoce (1ère phase en histopathologie) et les phénomènes inflammatoires (2ème phase) présents avant la quatrième semaine.

La tomodensitométrie a permis d'évaluer les modifications morphologiques précoces, avec des processus destructifs, développées au niveau des tissus profonds et notamment musculaires. Les lésions hypodenses (30 à $40 \mathrm{UH}$ ) en tomodensitométrie traduisent les mécanismes d'inflation hydrique (hydratation tissulaire) tel l'exsudat (1ère phase en histopathologie), alors que les hypodensités de densité pseudo-liquidienne (10 à $20 \mathrm{UH})$ notées à partir de $\mathrm{S}_{4}$ traduisent la présence de lésions nécrotiques (2ème phase). 
Des processus fibrotiques évolutifs plus tardifs sont responsables de l'atrophie du tissu musculaire. Il serait intéressant de suivre l'installation de la fibrose radioinduite dans cette cohorte d'animaux, morphologiquement par tomodensitométrie et par échographie, ainsi que l'apparition des phénomènes d'inflammation chronique, présents au niveau de la lésion par l'utilisation de la scintigraphie au ${ }^{67} \mathrm{Ga}$ (Lefaix et al., 1992b).

En résumé, ces deux méthodes d'imagerie permettent donc un diagnostic précoce des lésions et devraient permettre un suivi de ces lésions. La scintigraphie au ${ }^{67} \mathrm{Ga}$ apporte des informations fonctionnelles sur l'évolution des processus inflammatoires. La tomodensitométrie permet de visualiser des modifications précoces du tissu cutané et surtout des lésions profondes, notamment musculaires, en rapport avec une nécrose ischémique. Les résultats sont apparus homogènes et ont présenté une bonne corrélation avec les données histologiques.

Ces deux méthodes pourraient être proposées pour évaluer l'efficacité de traitement médico-chirurgical, au côté de l'IRM. En effet, il est envisageable de proposer la scintigraphie au ${ }^{67} \mathrm{Ga}$ dans l'évaluation expérimentale in vivo de l'efficacité pharmacologique de drogues anti-inflammatoires et également de nouvelles molécules, comme la superoxyde dismutase (Delanian et al., 1994 ; Lefaix et al., 1993a) ou la pentoxifylline (Dion et al., 1990 ; Lefaix et al., 1993b).

La tomodensitométrie devrait permettre d'évaluer l'importance des phénomènes destructifs affectant le tissu musculaire (fibrose, atrophie) de confirmer le retentissement de l'efficacité pharmacologique sur ces phénomènes et également de réaliser un suivi morphologique de la lésion d'irradiation avec définition de plusieurs caractères quantitatifs, comme les dimensions et la densité des lésions cutanées, sous-cutanées et musculaires.

Nous envisageons d'évaluer par la suite la gravité de la nécrose ischémique musculaire, à l'aide d'un nouveau radiopharmaceutique, le mifarmonab (Myoscint ${ }^{\circledR}$ - Centocor) qui utilise un fragment (Fab) d'anticorps monoclonal murin antimyosine spécifique marqué au chlorure d'Indium-111. Ce fragment se fixe électivement sur la myosine intracellulaire des myocytes nécrosés, car en cas d'atteintes cellulaires irréversibles provoquées par accident ischémique, la membrane cellulaire perd son intégrité et devient perméable au fragment d'anticorps mifarmonab. Le mifarmonab est actuellement utilisé chez l'homme, en cardiologie nucléaire, notamment pour évaluer l'importance de la nécrose myocardique, après infarctus; il pourrait de même contribuer à l'évaluation de l'étendue et de la gravité de la nécrose musculaire observée à la fin du premier mois après irradiation, dans notre modèle expérimental (Lefaix et al., 1992b, 1993b).

\section{Remerciements}

Ce travail a bénéficié du contrat RB96.12 du Comité de Radioprotection d’EDF. 


\section{RÉFÉRENCES}

Ando A., Nitta K., Ando I., Sanada S., Katsuda S., Tonami N., Hiraki T., Hisada K., Ogawa H. (1990) Mechanism of gallium 67 accumulation in inflammatory tissue. Eur. J. Nucl. Med., 17, 21-27.

Bitar R.A., Scheffel U., Murphy P.A., Bartlett J.G. (1986) Accumulation of indium-111labeled neutrophils and gallium-67 citrate in rabbit abscesses. J. Nucl. Med., 27, 1883-1889.

Brocheriou C., Verola O., Lefaix J-L., Daburon F. (1986) Histopathology of cutaneous and subcutaneous irradiation-induced injuries. Br. J. Radiol., 19 (Supp.) 101-104.

Camargo E.E., Wagner H.N., Tsan M.F. (1979). Studies on gallium accumulation in inflammatory lesions : IV. Kinetics of accumulation and role of polymorphonuclear leukocytes in the distribution of gallium in experimental inflammatory exudates. Nuklearmedizin, 18, 147-150.

Chalansonnet A., El Kamouni N., Briguet A., Daburon F., Lefaix J.-L. (1993) Mise en évidence des effets d'une irradiation aiguë localisée par imagerie de résonance magnétique du tissu cutané. Radioprotection, 28, 411-421.

Daburon F. (1986) Biophysical methods for assessing the radiation dose causing lesions in the skin and subcutaneous tissues. Brit. J. Radiol., 19 (Supp.) 75-82.

Daburon F., Lefaix J.L., Remy J., Bolnot D., Guilbaud J. (1986) Development of lesions resulting from acute localized irradiation in the pig: trials for medical and surgical management. Brit. J. Radiol., 19 (Supp.) 128-130

Delanian S., Lefaix J.L., Housset M. (1993) La fibrose iatrogénique en cancérologie (2ème partie) : principales étiologies et possibilités thérapeutiques. Bull. Cancer, 80, 202-212.

Delanian S., Baillet F., Huart J., Lefaix J.L., Molard C. (1994) Housset M. Successful treatment of radiation-induced fibrosis using liposomal $\mathrm{Cu} / \mathrm{Zn}$ superoxide dismutase : clinical trial. Radiother. Oncol., 32, 12-20.

Dion M.W., Hussey D.H., Doornbos J.F., Vigliotti A.P., Wen B.C., Anderson B. (1990) Preliminary results of a pilot study of pentoxifylline in the treatment of late radiation soft tissue necrosis. Int. J. Radiation Oncology Biol. Phys., 19, 401-407.

Gelrud L.G., Arseneau J.C., Milder M.S., Kramer R.J., Swann S.J., Cannelos G.P., Johnston G.S. (1974) The kinetics of gallium incorporation into inflammatory lesions : experimental and clinical studies. J. Lab. Clin. Med., 83, 489-495.

Hoffer P.B., Huberty J., Khayam-Bashi H. (1977) The association of ${ }^{67} \mathrm{Ga}$ and lactoferrin J. Nucl. Med., 18, 713-717.

Hoffer P.B. (1980) Gallium : mechanisms. J. Nucl. Med., 21, 282-285.

Hoffschir D., Toubeau M., Fayart G., Daburon F. (1989) Utilisation potentielle du ${ }^{201} \mathrm{Tl}$ pour l'évaluation scintigraphique des lésions d'irradiation : étude expérimentale sur le porc. J. Med. Nucl. Biophys., 13, 157-165.

Lefaix J-L., Verola O., Daburon F., Brocheriou C. (1985) Les lésions cutanées et musculaires après irradiation aiguë chez le porc. Etude histologique et histoenzymologique. Ann. Pathol., 5, 249-258.

Lefaix J.L., Daburon F., Fayart G. (1992a) Dosimetry study of an accidental overexposure to ${ }^{192}$ Ir gamma rays. Health Phys., 63, 692-694.

Lefaix J.L., Daburon F., Tricaud Y. (1992b) Évolution radiopathologique spontané et après traitement médical dans deux modèles d'accident d'irradiation localisée. Bull. Cancer/Radiothér., 79, 189-198.

Lefaix J.L., Delanian S., Leplat J.J., Tricaud Y., Martin M., Hoffschir D., Daburon F., Baillet F. (1993a) La fibrose cutanéo-musculaire radio-induite (III) : efficacité thérapeutique majeure de la superoxyde dismutase $\mathrm{Cu} / \mathrm{Zn}$ liposomiale. Bull. Cancer, 80 , $799-807$. 


\section{MISE EN ÉVIDENCE DES EFFETS D'UNE IRRADIATION AIGUË LOCALISÉE}

Lefaix J.L., Martin M., Tricaud Y., Daburon F. (1993b) Muscular fibrosis induced after pig skin irradiation with single doses of ${ }^{192} \mathrm{Ir} \gamma$-rays. Br. J. Radiol., 66, 537-544.

Menon S., Wagner H.N., Tsan M.F. (1978) Studies on gallium accumulation in inflammatory lesions : II. Uptake by staphylococcus aureus. J. Nucl. Med, 19, 44-47.

Nénot J.C. (1990) Medical and surgical management for localized radiation injuries. Int. I. Radiat. Biol., 57, 783-795

Raina S., Samuel A.M. (1992) Isotope angiography and blood pool imaging as a procedure for assessing radiation-induced injuries to the hands. Clin. Nucl. Med., 17, 646-651.

Tsan M.F., Chen W.Y., Scheffel U., Wagner H.N. (1978a) Studies on gallium accumulation in inflammatory lesions: I. Gallium uptake by human polymorphonuclear leukocytes. J. Nucl.. Med., 19, 36-43

Tsan M.F. (1978b) Studies on gallium accumulation in inflammatory lesions : III. Roles of polymorphonuclear leukocytes and bacteria. J. Nucl. Med., 19, 492-495

Tsan M.F. (1985) Mechanism of gallium 67 accumulation in inflammatory lesions. J. Nucl. Med., 26, 88-92

Verola O., Lefaix J-L., Daburon F., Brocheriou C. (1986) Vascular damage after acute local irradiation: a light and electron microscope study. Br. J. Radiol., 19 (Supp.) 104-108.

Weiner R., Hoffer P. B, Thakur M.L. (1981) Lactoferrin : its role as a Ga-67-binding protein in polymorphonuclear leukocytes. $J$. Nucl. Med., 22, 32-37. 